Ensino

\title{
Soma iterada de algarismos de um número racional
}

\author{
Digital root of a rational number
}

\author{
Eudes Antonio Costa'(i), Deyfila da Silva Lima'(i), \\ Élis Gardel da Costa Mesquita' ${ }^{(\infty)}$, Keidna Cristiane Oliveira Souza'(i)
}

'Universidade Federal do Tocantins, Palmas, TO, Brasil

\section{RESUMO}

A soma iterada de algarismos, $S^{*}(n)$, sendo $n$ um número inteiro positivo, é o algarismos $0 \leq b \leq 9$ obtido por meio de um processo iterativo da aplicação $S(n)$, a soma de algarismos (dígitos) do número $n$, em que cada iteração é obtida a partir do resultado anterior de modo a restar apenas o algarismo b. Por exemplo, a soma iterada de 999999 é igual a 9, pois 9+9+9+9+9+9=54 e 5+4=9, ou seja $S(999999)=54$ e $\mathrm{S}(54)=9$, assim $\mathrm{S}^{\star}(999999)=9$. A soma dos algarismos de um número inteiro positivo, e até a soma iterada de algarismos, é assunto recorrente em olímpiadas de matemática e foi abordado em diversos trabalhos, por exemplo em Ghannam(2012), Ismirli(2014) ou Lin(2016). Neste estendemos a aplicação S* a um número racional positivo x com representação decimal finita. Destacamos o seguinte resultado: dado um número racional positivo $x$, com representação decimal finita, e soma dos seus algarismos 9 , então quando $x$ é dividido por potências de 2, o número resultante também tem a soma dos seus algarismos igual a 9. Tal fato também ocorre quando o número $\mathrm{x}$ é dividido por potências de 5 . Resultados similares foram encontrados quando a soma dos algarismos de $x$ for 3 ou 6 .

Palavras-chave: Aplicação soma de algarismos; Congruência; Divisibilidade; Órbita e Soma iterada de algarismos

\section{ABSTRACT}

The digital roots $S^{*}(x)$, of a $n$ positive integer is the digit $0 \leq b \leq 9$ obtained through an iterative digit sum process, where each iteration is obtained from the previous result so that only the $b$ digit remains. For example, the iterated sum of 999999 is 9 because $9+9+9+9+9+9=54$ and $5+4=9$. The sum of the digits of a positive integer, and even the digital roots, is a recurring subject in mathematical competitions and has been addressed in several papers, for example in Ghannam (2012), Ismirli (2014) or Lin (2016). Here we extend the application $S^{\text {ast }}$ to a positive rational number $x$ with finite decimal representation. We highlight the following result: given a rational number $x$, with finite decimal representation, and the sum of its digits is 9 , so when divided $x$ by powers of 2 , the number resulting also has the sum of its digits 
9. Fact that also occurs when the $x$ number is divided by powers of 5 . Similar results were found when the $\mathrm{x}$ digit sum is 3 or 6 .

Keywords: Congruence; Digital root: Divisibility; Orbit and Sum of digits function

\section{INTRODUÇÃO}

Sabemos que a Matemática se desenvolveu por meio de tentativas em solucionar situações-problemas. São diversas as situações que motivaram e motivam matemáticos e aficionados a compreenderem e resolverem problemas em Matemática, por exemplo, podem ser, problemas cotidianos, problemas relacionados à fenômenos naturais ou curiosidades, entre outros. Dessa forma, vemos que o mistério ou desafio aguça a curiosidade humana e esta, por sua vez, pode ser um poderoso motivador para a aprendizagem em Matemática na obtenção de novos resultados. Algumas vezes, a generalização do resultado é motivado por um caso ou uma observação particular. Neste intuito consideremos com a seguinte questão desafio:

Problema 1. Considere o número $n=20202020 \ldots 2020$, em que 2020 repete 2020 vezes. Qual é a soma iterada de $n$ ?

Aqui denotamos por $\mathbb{Z}_{+}$o conjunto dos números inteiros positivos, o qual é equivalente ao conjunto dos números naturais $\mathbb{N}=\{0,1,2, \ldots n, \ldots\}$.A soma de algarismos de um número natural (inteiro positivo) $n \in \mathbb{Z}_{+}$é obtido pela adição dos algarismos deste número. Formalmente temos que

Definição 1. A aplicação soma de algarismos S é uma aplicação que a cada $n \in \mathbb{Z}_{+}$na forma

$$
n_{l} n_{l-1} \ldots n_{1} n_{0}, \operatorname{com} l \in \mathbb{N},
$$

associa ao número natural $\mathrm{m}$ dado por 
$S(n)=n_{l}+n_{l-1}+\cdots+n_{1}+n_{0}:=m$.

Exemplo 1. Segue da Definição 1 que $S(2020)=2+0+2+0=4$. Enquanto $S(123456)=1+2+3+4+5+6=21$, e $S(21)=S(S(123456))=3$.

No último caso, aplicamos $\mathrm{S}$ ao número $\mathrm{m}=\mathrm{S}(\mathrm{n})$, para $\mathrm{n}=123456$, chamamos este processo de iteração, e o conjunto de iterações de órbita. De um modo geral, temos

Definição 2. (a) Seja S a aplicação soma de algarismos do um número $n \in \mathbb{Z}_{+}$. As iterações de S são definidas por

$$
S^{1}(n)=S(n), S^{t}(n)=S\left(S^{t-1}(n)\right), t \leq 2 .
$$

(b) A órbita de um número $n \in \mathbb{Z}_{+}$, por $S$, denotado por $\mathcal{O}(n)$, é o conjunto $\left\{n, S,(n), S^{2}(n), \ldots, S^{k}(n), \ldots\right\}$.

Temos o seguinte resultado

Teorema 1. (Zeitz, 1999, Problem 7.2.11), (Izmirli, 2014, Theorem 1.1) Seja n um número inteiro positivo e $\mathrm{S}(\mathrm{n})$ denota a soma dos algarismos de $\mathrm{n}$. Num número finito de iterações, a órbita $\mathcal{O}(n)=\left\{n, S,(n), S^{2}(n), \ldots, S^{k}(n), \ldots\right\}$ se torna constante.

Segue do Teorema 1 que a órbita de n, ou seja, a sequência $\mathcal{O}(n)=\left\{S^{k}(n)\right\}_{k \in \mathbb{N}}$ se tornará constante a partir de um certo índice $k_{0}$. Neste caso, vamos denotar por $S^{*}(n)=S^{k_{0}}(n)$ o valor constante o qual denominamos soma iterada dos algarismos do número inteiro positivo $n$. E a demonstração encontra-se nas referências indicadas. Apresentemos agora a Resolução: do Problema 1.

Inicialmente veja que $S(2020)=4$. Para $n=20202020 \ldots 2020$, temos 


$$
\begin{aligned}
& S(n)=2020 \times S(2020) \\
& =4+4+\cdots+4_{2020 \text { vezes }} \\
& =2020 \times 4=8080 .
\end{aligned}
$$

Como $S(8080)=16$, segue que $S(16)=S^{*}(n)=7$

Como motivação, consideremos o seguinte problema desafio:

Problema 2. Considere o número racional positivo com representação decimal finita $x=2020,20202020 \ldots 2020$ com parte inteira 2020, e na parte decimal 2020 repete 2020 vezes. Qual é a soma iterada de x?

Neste contexto não podemos aplicar a aplicação $S^{*}$, nem mesmo $S$, aos números racionais $\mathrm{x}$.

Nesta nota estenderemos a definição da aplicação $S$ para um número racional positivo com representação decimal finita. Ademais apresentaremos alguns resultados de nosso estudo, aqui destacamos: dado um número racional positivo x, com representação decimal finita, e soma dos seus algarismos 9, então quando $x$ é dividido por potências de 2 , o número resultante também tem a soma dos seus algarismos igual a 9. Tal fato também ocorre quando o número $x$ é dividido por potências de 5 . Resultados similares foram encontrados quando a soma dos algarismos de $\mathrm{x}$ for 3 ou 6 .

\section{METODOLOGIA}

De acordo com Lakatos e Marconi (2017) este trabalho é uma pesquisa com característica de cunho qualitativo, iniciamos com uma pesquisa bibliográca (livros ou artigos) sobre o tema, por isso se caracteriza como uma busca ao corpo de produção científica do tema que apresenta o ( ou trata do) assunto em estudo. 
Após o levantamento da bibliografia, fizemos uma revisão teórica e crítica. Por fim nesse arcabouço conceitual apresentamos nosso resultado de estudo.

\section{A APLICAÇÃO $S^{*}$ EM $\mathbb{Z}_{+}$E ALGUMAS PROPRIEDADES}

Nesta seção listamos alguns resultados envolvendo a aplicação soma de algarismos de um número inteiro positivo, como estudo preliminar para definirmos a aplicação $S$ num número racional positivo.

Definição 3. (Lin, 2016) Para o inteiro $a \in\{1,2,3,4,5,6,7,8\}$ consideramos $\mathrm{S}(-a)=$ $\mathrm{S}(9-a)$.

Exemplo 2. Segue que $S(-2)=S(9-2)=S(7)=7$. Veja que -2 e 7 têm o mesmo resto 7 quando dividido por 9 em aritmética modular.

Observação 1. Em Ghannam (2012) sugere ignorar o sinal negativo do número inteiro e assim assume que $S(-51)=S(51)$, ou seja, para todo $n \in \mathbb{Z}_{+}$, temse que $S(-n)=S(|n|)$. Neste caso a aplicação $S$ (e portanto $S^{*}$ ) não faria distinção entre um número inteiro positivo e um número inteiro negativo.

Proposição 1. Para todo $n$ inteiro, temos:

(a) $S^{*}(n) \leq 0$;

(b) $S^{*}(n)=n$ se, e somente se, $n \in\{1,2,3,4,5,6,7,8,9\}$;

(c) $S^{*}(n)<|n|$ se, e somente se, $|n| \geq 10$;

$(d) S^{*}(-n) \equiv-S^{*}(n)(\bmod 9)$.

Proposição 2. Para quaisquer $m$ e $n$ inteiros positivos, temos:

(a) $S^{*}(n)=9$ se, e somente se, $n=9 m$;

(b) $S^{*}(n)=3$ se, somente se, $n=9 m+3$;

(c) $S^{*}(n)=6$ se, e somente se, $n=9 m+6$.

Proposição 3. Para quaisquer $\mathrm{m}$ e $\mathrm{n}$ inteiros, temos:

(a) $S^{*}(m+n)=S^{*}\left(S^{*}(m)+S^{*}(n)\right)$;

(b) $S^{*}(m-n) \equiv S^{*}(m)-S^{*}(n)(\bmod 9)$.

Proposição 4. Para quaisquer $m$ e $n$ inteiros positivos, temos que $S^{*}(m \times n)=$ $S^{*}\left(S^{*}(m) \times S^{*}(n)\right)$. 
Proposição 5. Para todo $n$ inteiro positivo, vale $S^{*}(n !)=9$ se, e somente se, $n \geq$ 6.

Proposição 6. Para quaisquer $\mathrm{m}$ e $\mathrm{n}$ inteiros positivos, vale $S^{*}\left(m^{n}\right) \equiv$ $S^{*}(m)^{n}(\bmod 9)$.

A demonstração, das Proposições 1 à 6, pode ser encontrada em Ghannam (2012), Izmirli(2014) ou Lin (2016).

Uma aplicação da soma iterada (e destes resultados acima) é a técnica conhecida como a prova dos nove. Essa fornece um critério para decidirmos se uma determinada operação de adição, subtração, multiplicão ou divisão entre números inteiros (postivivos) está correta ou não. A técnica consiste em verificar se a soma iterada das parcelas (ou fatores) é igual a soma iterada do resultado (soma ou produto). Vejamos, vamos considerar a adição dos números 123456 e 234567. Fazendo a soma iterada temos que $S^{*}(123456)=3, S^{*}(234567)=$ 9 e $S^{*}\left(S^{*}(123456)+S^{*}(234567)\right)=3$. Por outro lado, $123456+234567=358023$ e $S^{*}(358023)=3$. Isso indica que o cálculo (pode) está correto. Veja por exemplo Rodrigues (2004).

Ademais apresentamos um resultado mais geral que a Proposição 2.

Proposição 7. Para quaisquer $m$ e $n$ inteiros positivos. Se $m \equiv n \bmod 9$, então $S^{*}(m)=S^{*}(n)$.

Demonstração. Sem perca de generalidade admita que $m=9 q+$ $n$ para algum $q \in \mathbb{N}$. Assim

$$
\begin{gathered}
S^{*}(m)=S^{*}(9 q+n) \\
\text { Prop. } 3 \\
=S^{*}\left(S^{*}(9 q)+S^{*}(n)\right) \\
\text { Prop. } 2 \\
=S^{*}\left(9+S^{*}(n)\right) .
\end{gathered}
$$

Donde obtemos que $S^{*}(m)=S^{*}(n)$.

Usamos também a soma iterada de algarismos para obtermos alguns critérios de divisibilidade de um número inteiro. Por exemplo, um número inteiro 
é divisível por 3 ou 9 se, e somente se, a soma de seus algarismos é divisível por 3 ou 9, respectivamente. Para maiores detalhes, veja Domingues (2009).

Observação 2. Lembramos que várias classes de números especiais surgiram em decorrência da aplicação soma de algarismos.

Os exemplos mais comuns são:

- O número de Niven são aqueles divisíveis pela soma de seus algarismos. Por exemplo, 21, pois 21 é divisível por $3=2+1$. Um outro exemplo é o número 27.

- O número de Smith é um número composto para o qual a soma de seus algarismos é igual a soma dos algarismos na sua decomposição em fatores primos. Por exemplo, $27=333$ e $2+7=9$, assim como $3+3+3=9$.

O leitor que desejar obter mais informações a respeito destas classes de números, sugerimos Cooper(1993), Koninck, Doyon e Kátai (2003), Guy (2013), Mcdaniel (1987), Oltikar e Wayland (1983), Pickover (2001) ou Yates (1986) .

\section{A APLICAÇÃO $S^{*}$ EM $\mathbb{Q}_{+}^{f}$ E ALGUMAS PROPRIEDADES}

Denotamos por $\mathbb{Q}_{+}^{f}$ o conjunto de números racionais $x=\frac{a}{b}$ que possuem representação decimal finita, ou seja, escrevemos $x$ na forma

$$
x=x_{l} x_{l-1} \ldots x_{1} x_{0}, x_{-1} x_{-2} \ldots x_{-m} \text {, para l, m naturais. }
$$

Para quaisquer $a, b \in \mathbb{Z}_{+}, \operatorname{com} b \neq 0$.

Nosso intento é apresentar uma definição (e propriedades relacionadas) a aplicação $S^{*}$ para os números $x \in \mathbb{Q}_{+}^{f}$. Antes porém, apresentamos um resultado que faremos uso.

Proposição 8. Para todo $n$ inteiro positivo da forma $n=10^{k}, k$ natural, segue que $S^{*}(n)=1$.

Demonstração. Aplicar indução em $k$ natural e Proposição 4.

O próximo resultado segue imediatamente das Proposições 4 e 8 . 
Proposição 9. Para todo $\mathrm{n}$ inteiro positivo tem-se $S^{*}\left(n \times 10^{k}\right)=$ $S^{*}(n)$, para cada $k \in \mathbb{N}$.

Um fato conhecido acerca dos números racionais que possuem representação decimal finita é o seguinte

Teorema 2. (Domingues, 2009) Um número racional $x=\frac{a}{b}$ admite representação decimal finita se, e somente se, quando escrito na forma irredutível, a decomposição em fatores primos de seu denominador possui apenas os fatores 2 ou 5.

Como consequência temos o seguinte

Corolário 1. (Domingues, 2009) Um número racional x admite representação decimal finita na forma $x_{l} x_{l-1} \ldots x_{1} x_{0}, x_{-1} x_{-2} \ldots x_{-m}$, para I, $\mathrm{m}$ naturais se, e somente se, $x=\frac{x_{l} x_{l-1} \ldots x_{1} x_{0}, x_{-1} x_{-2} \ldots x_{-m}}{10^{m}}$.

Agora estamos em condições de definir ou estender a aplicação soma de algarismos para um número racional que admite representação decimal finita, da seguinte forma

Definição 4. A aplicação soma de algarismos S é uma aplicação que a cada $x \in \mathbb{Q}_{+}^{f}$ associa ao número racional positivo $y=\frac{s(a)}{S(b)}$.

Exemplo 3. O número racional com representação decimal finita $x=2019$; 2020, conforme Corolário 1 pode ser escrito na forma $x=\frac{20192020}{10^{4}}$. Assim $S=(2019,2020)=\frac{S(20192020)}{S\left(10^{4}\right)}$. Segue da Proposição 8 que $S\left(10^{4}\right)=1$. Assim

$$
S(2019,2020)=\frac{S(20192020)}{S\left(10^{4}\right)}=\frac{2+0+1+9+2+0+2+0}{1}=16 .
$$

Teorema 3. Seja x um número racional positivo com representação decimal finita e $S(x)$ denote a soma dos algarismos de $x$.

Num número finito de iterações, a órbita $\mathcal{O}(n)=\left\{x, S(x), S^{2}(x), \ldots S^{k}(x), \ldots\right\}$ se torna constante. 
Demonstração. Basta observar que $S(x)$ é um número racional positivo com denominador 1 , ou seja, $S(x) \in \mathbb{Z}_{+}$. Agora aplique o Teorema 1 a órbita $\left\{S(x), S^{2}(x), \ldots, S^{k}(x), \ldots\right\}$.

Segue do Teorema 3 que a órbita de $x$, ou seja, a sequência $\left\{S^{k}(x)\right\}_{k \in \mathbb{N}}$ se tornará constante a partir de um certo índice $k_{0}$. Neste caso, também vamos denotar por $S^{*}(x)=S^{k_{0}}(x)$ o valor constante o qual (também) denominamos soma iterada de algarismos do número racional positivo x com representação decimal finita.

Exemplo 4. Dado o número racional $x=2019 ; 2020$, segue do Teorema 3 que $S^{*}(2019 ; 2020)=7$.

Observação 3. Dado o número $x \in \mathbb{Q}_{+}^{f}$, como $x=\frac{x_{l} x_{l+1} \ldots x_{1} x_{0} x_{-1} x_{-2} \ldots x_{-m}}{10^{m}}$, de uma forma direta, podemos considerar

$$
\begin{gathered}
S\left(x_{l} x_{l-1} \ldots x_{1} x_{0}, x_{-1} x_{-2} \ldots x_{-m}\right)=x_{l}+x_{l-1}+\cdots+x_{1}+x_{0}+x_{-1}+x_{-2}+\cdots+x_{-m} \\
=S\left(x_{l} x_{l-1} \ldots x_{1} x_{0} x_{-1} x_{-2} \ldots x_{-m}\right) .
\end{gathered}
$$

Agora estamos em condições de apresentar a Resolução: do Problema 2 .

Sabemos que $S(2020)=4$. Para $x=2020,20202020 \ldots 2020$, temos que

$$
\begin{gathered}
S(x)=2021 \times S(2020) \\
=4+4+\cdots+4(2021 \text { vezes }) \\
=2021 \times 4=8084 .
\end{gathered}
$$

Como $S(8084)=20$, segue que $S(20)=S^{*}(x)=2$.

Outras propriedades da aplicação soma dos algarismos.

Proposição 10. Seja $x=x_{l} x_{l-1} \ldots x_{1} x_{0}$ um inteiro positivo. Se $S^{*}(x)=9$, então $S^{*}=\left(\frac{x}{2^{n}}\right)=9$, para todo $n$ natural.

Demonstração. Temos que, 


$$
x=x_{l} x_{l-1} \ldots x_{1} x_{0}
$$

com $0 \leq x_{l} ; \ldots ; x_{0}<10$ e $x_{l} \neq 0$ : Fixamos $\mathrm{n}$ natural e multiplicamos a equação (1) por $\frac{1}{2^{n}}$; obtemos

$$
\begin{array}{r}
\frac{x}{2^{n}}=\frac{x_{l} x_{l-1} \ldots x_{1} x_{0}}{2^{n}} \\
=\frac{\left(x_{l} x_{l-1} \ldots x_{1} x_{0}\right) \times 5^{n}}{2^{n} 5^{n}} \\
=\frac{\left(x_{l} x_{l-1} \ldots x_{1} x_{0}\right) \times 5^{n}}{10^{n}}
\end{array}
$$

Então, conforme Definição 4

$$
\begin{aligned}
s\left(\frac{x}{2^{n}}\right) & =\frac{S\left(\left(x_{l} x_{l-1} \ldots x_{1} x_{0}\right) \times 5^{n}\right)}{S\left(10^{n}\right)} \\
& =\frac{S\left(\left(x_{l} x_{l-1} \ldots x_{1} x_{0}\right) \times 5^{n}\right)}{1} .
\end{aligned}
$$

Assim, é suficiente analisarmos a expressão $t=\left(x_{l} x_{l-1} \ldots x_{1} x_{0}\right)$. Como $S^{*}(x)=9$, segue que $x=9 q$ para algum natural $\mathrm{q}$ assim temos que $t=9 \mathrm{~m}$, fazendo $m=q \times 5^{n}$. Agora segue da Proposição 2 que $S^{*}(t)=9$. Donde concluímos o resultado.

Exemplo 5. Veja que $S^{*}(3 ; 6)=3+6=9$, bem como

$S^{*}(11 ; 25)=1+1+2+5=9$.

O próximo resultado é imediato

Corolário 2. Seja $x=x_{l} x_{l-1} \ldots x_{1} x_{0}, x_{-1} \ldots x_{-m} \in \mathbb{Q}_{+}^{f}$. Se $S^{*}(x)=9$, então $S^{*}\left(\frac{x}{2^{n}}\right)=$ 9, para todo $n$ natural 
De modo similar a Proposição 10 e Corolário 2 temos que

Proposição 11. Seja $x=x_{l} x_{l-1} \ldots x_{1} x_{0}$ um inteiro positivo. Se $S(x)=9$ então $S^{*}\left(\frac{x}{5^{n}}\right)=9$, para todo $n$ natural.

Corolário 3. Seja $x=x_{l} x_{l-1} \ldots x_{1} x_{0} ; x_{-1} \ldots x_{-m} \in \mathbb{Q}_{+}^{f}$. Se $S^{*}(x)=9$, então $S^{*}\left(\frac{x}{5^{n}}\right)$, para todo $n$ natural.

Proposição 12. Seja $x=x_{l} x_{l-1} \ldots x_{1} x_{0}$ um inteiro positivo. Se $S^{*}(x)=3$, então $S^{*}\left(\frac{x}{2^{n}}\right)=\left\{\begin{array}{c}3, \text { se } n \text { é } p a r, \\ 6, \text { se } n \text { é ímpar. }\end{array}\right.$

Demonstração. De modo similar a Proposição 10, segue que

$$
\begin{gathered}
S^{*}\left(\frac{x}{2^{n}}\right)=S^{*}\left(x \times 5^{n}\right) \\
=S^{*}\left(S^{*}(x)\right. \\
\times S^{*}\left(5^{n)}\right) \\
=S^{*}\left(3 \times S^{*}\left(5^{n}\right)\right) .
\end{gathered}
$$

O resultado segue do fato que $S^{*}\left(5^{n}\right)=1 ; 7$ ou 4 se $n$ é par, e $S^{*}\left(5^{n}\right)=5 ; 8$ ou 2 se $n$ é ímpar.

Donde segue que

Corolário 4. Seja $x=x_{l} x_{l-1} \ldots x_{1} x_{0}, x_{-1} \ldots x_{-m} \in \mathbb{Q}_{+}^{f}$. Se $S^{*}(x)=3$, então $S^{*}\left(\frac{x}{2^{n}}\right)=$ $\left\{\begin{array}{c}3, \text { se } n \text { é par, } \\ 6 \text {, se } n \text { é ímpar. }\end{array}\right.$

Análogo a Proposição 12 e Corolário 4 , obtemos:

Proposição 13. Seja $x=x_{l} x_{l-1} \ldots x_{1} x_{0}$ um inteiro positivo. Se $S^{*}(x)=6$, então $S^{*}\left(\frac{x}{2^{n}}\right)=\left\{\begin{array}{c}3, \text { se } n \text { é ímpar, } \\ 6, \text { se } n \text { é par. }\end{array}\right.$

Corolário 5. Seja $x=x_{l} x_{l-1} \ldots x_{1} x_{0}, x_{-1} \ldots x_{-m} \in \mathbb{Q}_{+}^{f}$. Se $S^{*}(x)=6$, então $S^{*}\left(\frac{x}{2^{n}}\right)=$ $\{3$, se $n$ é ímpar, \{, se $n$ é par. 


\section{ALGUNS PROBLEMAS}

Problema 3 (BQ-OBMEP 2008). Quantos números $n$ de 4 algarismos existem tais que $S(n)$ é maior que 34 ?

Problema 4 (OBM 2014). O número 2014 tem quatro algarismos distintos e $S(2014)=7$. Quantos números inteiros positivos têm essas duas propriedades?

Problema 5 (BQ-OBMEP 2009). (a) Listemos os primeiros 20092009 números naturais. Em seguida, na linha abaixo, substituímos, sucessivamente, cada número pela soma dos seus algarismos, até obtermos uma lista de números com apenas um algarismo. A lista tem mais algarismos 4 ou 5 ? Quantos 9 tem a lista?

(b) Determine $S^{*}\left(3^{2009}\right)$ e $S^{*}\left(17^{2009}\right)$.

Problema 6. (Pereira) $\mathrm{O}$ inteiro positivo $n$ é formado de $k$ algarismos 9. Mostre que $S\left(n^{2}\right)=9 k$ e $S^{*}\left(n^{2}\right)=9$.

Problema 7. (IMO 1975) Seja $S(n)$ a soma dos algarismos de $n$. Se $n=$ $4444^{4444}, a=S(n) e b=S(a)$. Quanto vale $S(b)$ ?

Problema 8. (OBM 2014) Encontre todos os inteiros $n, n>1$, com a seguinte propriedade: para todo $k, 0 \leq k<n$, existe $m$ um múltiplo de $n$, tal que $S(m)$ deixa resto $k$ na divisão por $n$.

Problema 9. (OBM 2018) Determine todos os pares ( $a$; b) de inteiros positivos para os quais $S(a n+b)-S(n)$ assume um número finito de valores ao variar $n$ nos inteiros positivos.

Problema 10. (Zeitz, 1999, Problem 7.2.11.b) Mostre que $S^{*}(a \times b)=8$, sendo a e b primos gêmeos, diferentes de 3 e 5 .

Problema 11. (Voight, Proposition 14) Considere $n>6$ um número perfeito par, então $S^{*}(n)=1$.

Problema 12. Seja $x_{4}$ um número inteiro positivo constituído de 4 algarismos, isto é, um número do tipo $x_{4}=a_{3} a_{2} a_{1} a_{0}$.Considere o seguinte algoritmo, que retorna o número $B \neq 0$, número Mágico de Ball, veja Costa e Mesquita (2014).

1. Dado $x_{4}$, com $a_{3} \neq a_{0}$ ou $a_{3}=a_{0}$ e $a_{2} \neq a_{1}$; 
2. Escreva o número $x_{4}^{\prime}$ com ordem inversa dos algarismos, ou seja, $x_{4}^{\prime}=$ $a_{0} a_{1} a_{2} a_{3}$

3. Encontre a diferença $y_{4}=\left|x_{4}-x_{4}^{\prime}\right|=b_{3} b_{2} b_{1} b_{0}$ (O qual deve-se considerar como um número de 4 algarismos, mesmo quando o algarismo $b_{3}$ for zero.);

4. Escreva o número $y_{4}^{\prime}=b_{0} b_{1} b_{2} b_{3}$;

5. Escreva o número $B=y_{4}+y_{4}^{\prime}$.

Mostre que para qualquer $x_{4}$, tem-se que $S^{*}(B)=9$.

\section{RESULTADOS E DISCUSSÃO}

Segundo Meyer (2018), Nikola Tesla (1856-1943) é uma das mentes mais geniais do final do século XIX e início do século XX, cujas contribuições para as ciências se deram no campo do eletromagnetismo e mecânica, observou que ao dividirmos ou multiplicarmos consecutivamente por 2 uma circunferência, os ângulos (medido em grau) resultantes têm a propriedade de que a soma dos algarismos é igual (sempre) a 9, por exemplo $S(360)=S(180)=S(90)=S(45)=$ $S(22 ; 5)=9$. Alguns vídeos no youtube, basta fazer uma busca por Nikola Tesla, exibem essa observação de Tesla e apregoam um misticismo associado à tal fato, atribuem ainda a ele a seguinte enigmática sentença "Se você soubesse da magnificência dos números 3, 6 e 9, então você teria a chave do Universo." Aqui mostramos que tal fato, soma dos algarismos 3, 6 ou 9 e divisão por 2 ou 5, é uma propriedade aritmética e apenas uma aplicação de $S^{*}$ a um x número inteiro ou racional positivo com representação decimal finita.

Intrigados com as afirmações atribuídos a Tesla, fizermos este primeiro estudo para mostrar as propriedades aritméticas envolvidas. Com este pretendemos iniciar uma discussão sobre a "função" soma dos algarismos nos números racionais, que quase nada sabemos por inexistência de trabalho similar ao nosso (desconhecemos ou não achamos em nenhuma base de dados). Algumas questões ainda nos provoca: Como estender para qualquer racional? É possível estender para todo número real? 


\section{REFERÊNCIAS}

Cooper, C. N.; Kennedy, Robert E. On consecutive Niven numbers. Fibonacci Quart, volume 21, $1993(146-151)$.

Costa, Eudes A.; Mesquita, Élis Gardel da C. O Número Mágico M. Revista da Olimpíada, volume 9, 2014 (33-43).

Domingues, Hygino Hugueros. Fundamentos de aritmética. Editora da UFSC, 2009.

Ghannam, Talal. The Mystery of Numbers: Revealed Through their Digital Root . Talal Ghannam publisher. 2012.

Guy, Richard. Unsolved problems in number theory. Springer Science \& Business Media. 2013

Izmirli, Ilhan M. On Some Properties of Digital Roots. Advances in Pure Mathematics, volume 4, 06, 2014 (295).

Koninck, Jean-Marie; Doyon, Nicolas; Kátai, Imre. On the counting function for the Niven numbers. Acta Arithmetica, volume 106, 03, 2003 (265-275).

Lakatos, E. M.; Marconi, M. A. Fundamentos de Metodologia Científica. São Paulo: Atlas Editora, 2017.

Lin, Chia-Yu. Digital root patterns of three-dimensional space Recreational Mathematics Magazine, volume 3, 05, 2016 (9-31).

Meyer, Maximiliano. Nikola Tesla, o gênio mais injustiçado da história. Oficina da Net. https://www.oficinadanet.com.br/post/14537-nikola-tesla-o-genio-mais-injusticadoda- história « acesso em 07 nov. 2018 »

McDaniel, Wayne L. The existence of infinitely many k-Smith numbers. Fibonacci Quart, volume 25,1987 (76-80).

Oltikar, Sham; Wayland, Keith. Construction of Smith numbers . Mathematics Magazine, volume 56, 1, 1983 (36-37).

Pereira, Valberto R. F.. Domingo regado a Repunits. Eureka (SBM-OBM), volume 29, 2009. www.obm.org.br

Pickover, C. A. A Brief History of Smith Numbers. Wonders of Numbers: Adventures in Mathematics, Mind, and Meaning, volume56, 104, 1983 (247-248).

Rodrigues, Flávio Wagner. A prova dos nove. Revista do Professor de Matetática, 14, 2004 (1$3)$. 
Voight, John. Perfect numbers: an elementary introduction. University of California journal Berkley, 1998.

Yates, Samuel. Special sets of Smith numbers. Mathematics Magazine, volume 59, 05, 1986 (293-296).

Zeitz, Paul. The art and craft of problem solving. John Wiley New York publisher. 1999.

\section{Contribuição dos Autores}

\section{1 - Eudes Antonio Costa}

Professor Adjunto; Doutor em Matemática

https://orcid.org/0000-0001-6684-9961 e eudes@uft.edu.br

Contribuição: Writing - original draft

\section{2 - Deyfila da Silva Lima}

Professora temporária, Mestranda

https://orcid.org/0000-0001-8028-9323 e deyfila@uft.edu.br

Contribuição: Writing - original draft

\section{3 - Élis Gardel da Costa Mesquita}

Professor Adjunto; Doutor em Matemática

https://orcid.org/0000-0003-2385-4108 e elisgardel@uft.edu.br

Contribuição: Writing - original draft

\section{4 - Keidna Cristiane Oliveira Souza}

Professora Adjunta; Doutora em Matemática https://orcid.org/0000-0001-8404-7380 e keidna@uft.edu.br

Contribuição: Writing - original draft 